\title{
Teenagers, adolescents, endometriosis and recurrence: a retrospective analysis of recurrence following primary operative laparoscopy
}

\author{
Emmanuel Kalu • William McAuley • \\ Robert Richardson
}

Received: 3 January 2008 / Accepted: 7 February 2008/Published online: 21 March 2008

(C) Springer-Verlag 2008

\begin{abstract}
In this paper, we aim to describe the clinical features and treatment outcome following the laparoscopic treatment of endometriosis in teenagers and adolescents. This is a retrospective review of girls aged 21 and below who underwent operative laparoscopy for endometriosis. We identified two groups: (1) the EMAT group, being those who underwent their primary laparoscopic surgery under the Endometriosis and Minimal Access Therapy team (EMAT), and (2) the non-EMAT group, consisting of those referred to the EMAT team with recurrent symptoms following primary operative laparoscopy by other gynaecologists. Symptom recurrence and the need for re-operation was compared between the two groups. Twenty-eight girls, age range 15-21 years, were identified. All had pelvic pain unresponsive to the pill and non-steroidal anti-inflammatory drugs (NSAIDs). Most disease was atypical, with the uterosacral ligaments and ovarian fossae being the most common sites. The re-operation rate per surgical team at 24 months was $14.2 \%$ for the EMAT group vs. $42.8 \%$ for the non-EMAT group. All recurrences in the non-EMAT group coincided with the location of primary disease. Disease recurrence occurred most commonly in the uterosacral ligaments. Endometriosis in teenagers is mainly atypical and, in our series, commonly affects the uterosacral ligaments. Disease in this location is deeply infiltrating and is usually not amenable to electrocoagulation. We advocate disease resection to minimise recurrence.
\end{abstract}

E. Kalu $(\bowtie) \cdot$ W. McAuley $\cdot$ R. Richardson

Gynaecology, Chelsea and Westminster Hospital,

369 Fulham Road,

London SW10 9NH, UK

e-mail: ekalu@doctors.org.uk
Keywords Teenagers · Adolescents · Endometriosis · Operative laparoscopy $\cdot$ Recurrence

\section{Introduction}

Endometriosis (the presence of functional endometrial glands and stroma outside the uterine cavity) can strike at any age. The true incidence of this disease among teenagers and adolescents remains unknown. It has been identified in over $50 \%$ of teenagers with chronic pelvic pain and dyspareunia $[1,2]$ and in about $70 \%$ of teenagers with pelvic pain not responding to non-steroidal anti-inflammatory drugs (NSAIDs) and oral contraceptive pills [3]. Severe dysmenorrhoea and pelvic pain, two major symptoms of endometriosis, remain the single most frequent causes of absenteeism from school [4] and account for the loss of over 140 million work hours yearly [5]. The treatment of this often chronic and debilitating disease when found is, therefore, of prime importance in young people. Although various authors have characterised endometriosis in teenagers and adolescents [1-3], information on treatment outcome in this age group is limited. In this retrospective cohort study, we describe the clinical features and treatment outcome of teenagers and adolescents who underwent the laparoscopic treatment of endometriosis at the Chelsea and Westminster Hospital, London.

\section{Methods}

Teenagers and adolescents, defined as those patients aged 21 years or less at the time of primary surgery, who had had operative laparoscopy were identified from the hospital database. Patients were divided into two groups: (1) the 
EMAT group, being those who underwent their primary laparoscopic surgery under the (specialist) Endometriosis and Minimal Access Therapy team (EMAT), and (2) the non-EMAT group, consisting of those referred to the EMAT team with recurrent symptoms following primary operative laparoscopy by other gynaecologists. Follow up to 24 months was completed. Treatment outcome was assessed on the basis of symptom recurrence and the need for second operative laparoscopy. All of the patients were seen in the EMAT clinic following referral by the nonEMAT team or as routine follow up after primary surgery by the EMAT team, during which, assessment for the need for further surgery was made. The decision for re-operation was based on the presence of persistent symptoms, high pain score, previous non-response to medical therapy and patients' consent. All second surgeries were performed by the EMAT team. During repeat laparoscopy, the index disease location was compared to the disease location at the time of primary surgery. Disease recurrence, as well as the site of recurrence, was compared between the two groups. The results were analysed using the Statistical Package for the Social Sciences (SPSS, Surrey, UK). With an alpha value of 0.05 , the power of the study (type 2 error) to detect a significant difference in disease recurrence between the two groups would be $85.3 \%$.

\section{Results}

Using the hospital coding system, we identified 570 women who underwent laparoscopic treatment of endometriosis at the Chelsea and Westminster Hospital, London, between 2000 and 2005. A total of $4.9 \%$ (28/570) were teenagers and adolescents, age range 15-21 years. All had pelvic pain unresponsive to the pill and NSAIDs. The nature of the pelvic pain was cyclical in $78 \%$ and non-cyclical in $21 \%$ of the cases. Other presenting symptoms and their frequencies are shown in Table 1. Fifty percent of the primary laparoscopic procedures (14/28) were performed by the EMAT team, and the other half by non-EMAT consultants, including referrals from secondary care. Disease severity was equally distributed between the two groups. In the nonEMAT group, treatment was exclusively by electrocoagulation/ablation. In contrast, in the EMAT group, treatment was predominantly by lesion resection. The uterosacral ligaments (USL) and ovarian fossae were the most common sites affected (Fig. 1). Atypical endometriosis constituted the most common lesions in this age group (Fig. 2 and Picture 1). The re-operation rate at 2 years was $14.2 \%$ $(2 / 14)$ for the EMAT group vs. $42.8 \%(6 / 14)$ for the nonEMAT group ( $p=0.023$ ) (Figs. 3 and 4$)$.
Table 1 Presenting symptoms in teenagers and adolescents with endometriosis

\begin{tabular}{lcc}
\hline Symptoms & Total number & Percentage $^{\mathrm{a}}$ \\
\hline Pelvic pain & $28 / 28$ & $100 \%$ \\
-Cyclical & $22 / 28$ & $78.57 \%$ \\
-Non-cyclical & $6 / 28$ & $21.42 \%$ \\
Dyspareunia & $17 / 23$ & $73.39 \%$ \\
Irregular periods & $13 / 28$ & $46.45 \%$ \\
Vaginal discharge & $3 / 28$ & $10.71 \%$ \\
Bowel symptoms & $3 / 28$ & $10.71 \%$ \\
Urinary symptoms & $1 / 28$ & $3.57 \%$ \\
\hline
\end{tabular}

${ }^{a}$ The percentages do not add up to $100 \%$ because the patients often had more than one symptom

${ }^{\mathrm{b}}$ Five teenagers were not sexually active

Disease recurrence occurred most commonly in the uterosacral ligaments (Fig. 3). All recurrences in the nonEMAT group coincided with the location of primary disease.

\section{Discussion}

About $5 \%$ of women who underwent surgery for endometriosis in the 5-year period were teenagers/adolescents. This equates to about five young women per annum, a small but significant number. The chronic debilitation associated with this disease makes its management in these young people of prime importance. Although laparoscopy has been shown to be safe, minimally invasive and enables a definitive diagnosis and treatment of endometriosis in adolescents [6], the decision to subject these young people to surgery is often delayed and preceded by a prolonged period of unsuccessful symptomatic treatment. Surgical treatment,

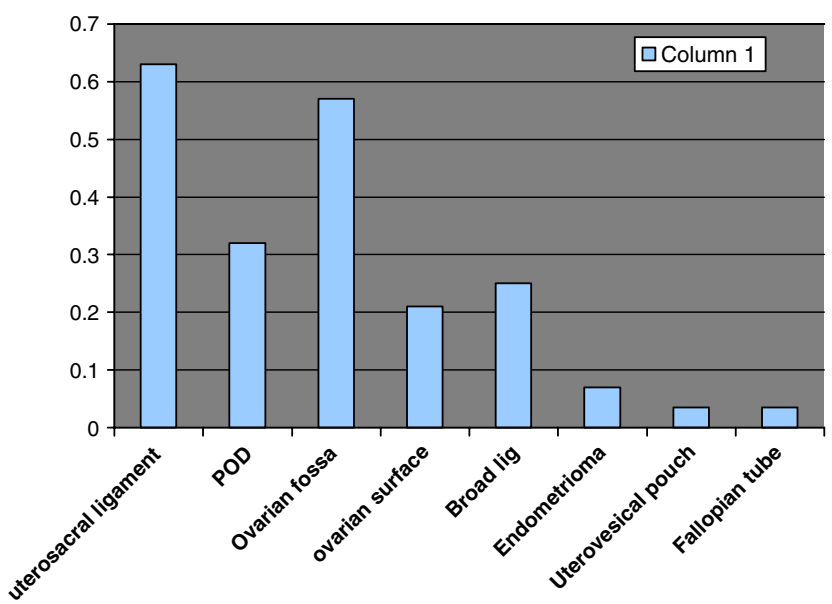

Fig. 1 Disease location 
Fig. 2 Types of lesions found in teenagers/adolescents

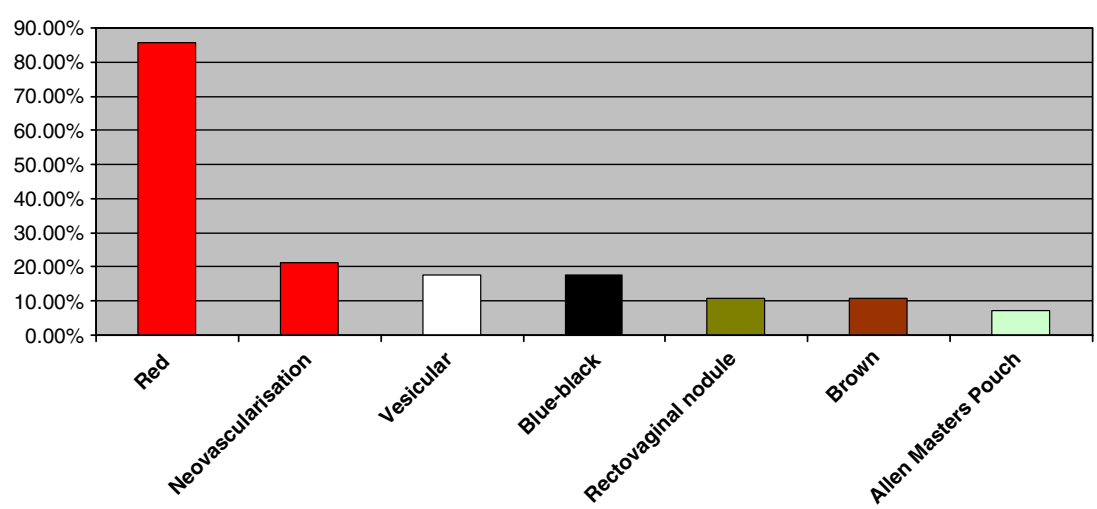

however, is associated with recurrence, and this seems to depend on who operates.

In our series, girls who were operated by generalist gynaecologists had a three-fold increase in symptom recurrence and the need for re-operation, compared to their counterparts, who were operated by an endometriosis specialist team. This difference in treatment outcome may be related to the mode of surgical treatment. While the treatment was exclusively by electrocoagulation/ablation in the non-EMAT group, in the EMAT group, treatment was predominantly by lesion resection. This is important, since the most common location of endometriosis in this series were the uterosacral ligaments. Disease in this location is usually deeply infiltrating and not amenable to electrocoagulation [7]. The high recurrence in the non-EMAT group may, therefore, be related to the incomplete ablation of deeply infiltrating disease. The fact that there was always recurrence in the areas of previous ablation in the non-EMAT group cases also points to insufficient primary treatment in this group.

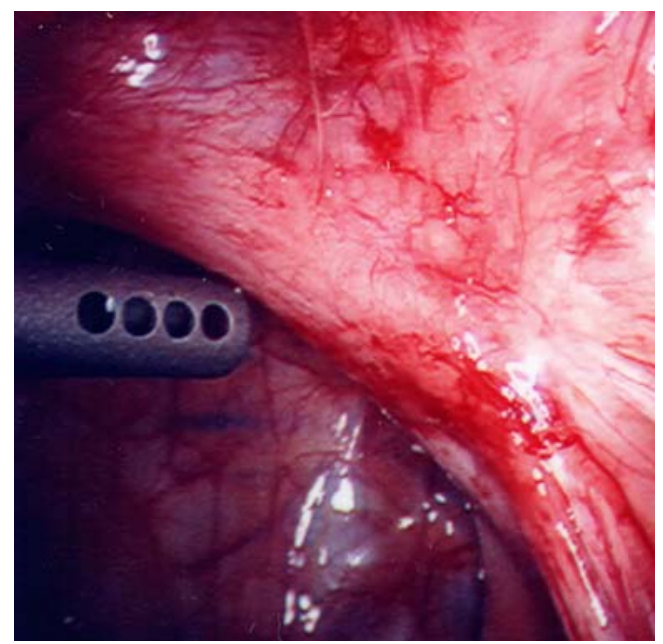

Picture 1 Red endometriosis in the uterosacral ligament
Following disease resection by the specialist EMAT team, symptom recurrence after 2 years was $14 \%$. This is similar to other published series following the resection of teenage endometriosis by a specialist team $[8,9]$. In their series, Stavroulis et al. [8] reported that eight of 11 teenagers diagnosed with endometriosis were completely pain-free or greatly improved and a further two reported partial improvement following laparoscopic resection.

Like other researchers $[2,10]$, we found red endometriosis with neovascularisation to be the most frequent type of lesion in these young women. Red lesions include at least three morphologic forms, red polyps, red vesicles and red flame-like lesions [11], and they are thought to be more biologically active than their brown/black counterparts and more strongly associated with dysmenorrhoea [12]. The typical blue-black (powder burn) implants common in adult women were present in only $18 \%$ of our cases. Other subtle appearances of endometriosis that may easily go unrecognised during surgery in young women include vesicular lesions and lesions in peritoneal pockets. The role that atypical disease plays in the recurrence/persistence of symptoms in this age group remains uncertain. It is possible that failure to recognise and treat atypical disease may also contribute to the non-resolution of symptoms.

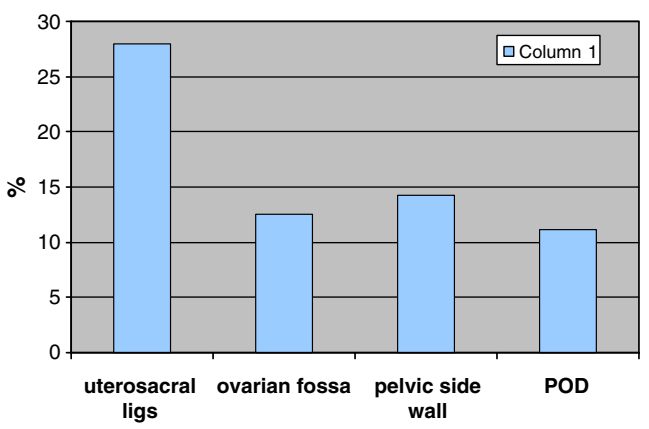

Fig. 3 Site of disease recurrence 
Recurrence-free interval after primary

Surgery for endometriosis in young adults graph

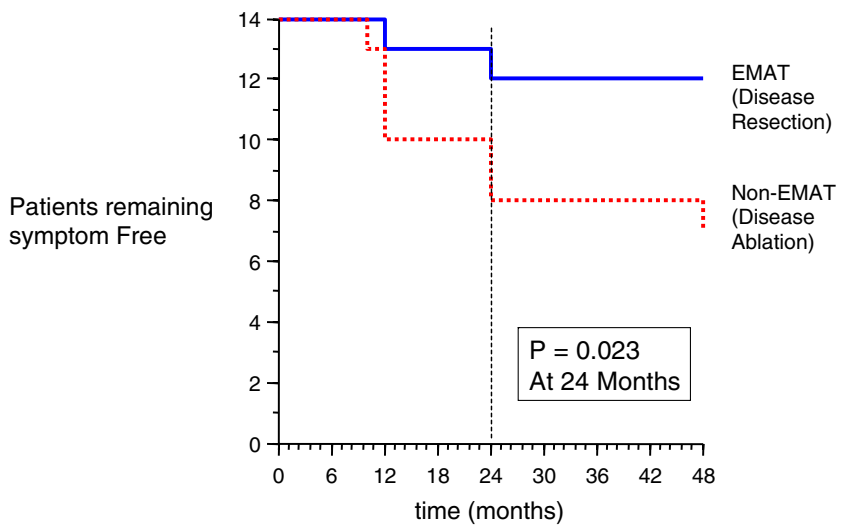

Fig. 4 Recurrence-free interval after primarysurgery for endometriosis in young adults

However, because there was a $100 \%$ recurrence in the areas of previous ablation in our series, the persistence of symptoms in the non-EMAT group is more likely due to incomplete treatment by ablation.

\section{Conclusion}

Endometriosis in teenagers is mainly atypical, and, in our series, commonly affects the uterosacral ligaments. As disease in this area is usually not amenable to electrocoagulation/ablation, we recommend disease resection to minimise recurrence. Since resection may impose greater surgical risks, we suggest that primary surgery in these patients should be within a centre where disease resection can be performed.

\section{References}

1. Goldstein DP, deCholnoky C, Emans SJ, Leventhal JM (1980) Laparoscopy in the diagnosis and management of pelvic pain in adolescents. J Reprod Med 24:251-256

2. Reese KA, Reddy S, Rock JA (1996) Endometriosis in an adolescent population: the Emory experience. J Pediatr Adolesc Gynecol 9:125-128

3. Laufer MR, Goltein L, Bush M, Cramer DW, Emans SJ (1997) Prevalence of endometriosis in adolescent girls with chronic pelvic pain not responding to conventional therapy. J Pediatr Adolesc Gynecol 10:199-202

4. Lamb EJ (1981) Clinical features of primary dysmenorrhea. In: Dawood MY (ed) Dysmenorrhea. Williams \& Wilkins, Baltimore, pp 107-129

5. Ylikorkala O, Dawood MY (1978) New concepts in dysmenorrhea. Am J Obstet Gynecol 130:833-847

6. Wolfman W, Kreutner K (1984) Laparoscopy in children and adolescents. J Adolesc Health Care 5:261-265

7. Hasson HM (1979) Electrocoagulation of pelvic endometriotic lesions with laparoscopic control. Am J Obstet Gynecol 135:115-121

8. Stavroulis AI, Saridogan E, Creighton SM, Cutner AS (2006) Laparoscopic treatment of endometriosis in teenagers. Eur J Obstet Gynecol Reprod Biol 125:248-250

9. Marsh EE, Laufer MR (2005) Endometriosis in premenarcheal girls who do not have an associated obstructive anomaly. Fertil Steril 83:758-760

10. Laufer MR, Sanfilippo J, Rose G (2003) Adolescent endometriosis: diagnosis and treatment approaches. J Pediatr Adolesc Gynecol 1693(3 Suppl):S3-S11

11. Jensen RPS, Russell P (1986) Nonpigmented endometriosis: clinical, laparoscopic, and pathologic definition. Am J Obstet Gynecol 155:1154-1159

12. Vernon MW, Beard JS, Graves K, Wilson EA (1986) Classification of endometriotic implants by morphologic appearance and capacity to synthesize prostaglandin F. Fertil Steril 46:801-806 